\title{
Comparative study of tissue reactivity to n-butyl-2-cyanoacrylate and nylon monofilament thread on pericranium-cutaneous flaps in rats ${ }^{1}$
}

\author{
William César Cavazana', Sergio Osamu Ioshii', Roberto Kenji Nakamura Cuman ${ }^{\mathrm{III}}$, Luis Augusto Passeri ${ }^{\mathrm{IV}}$ \\ DOI: http://dx.doi.org/10.1590/S0102-86502014000400007 \\ IFellow PhD degree, Department of Surgery, Faculty of Medical Sciences, State University of Campinas (UNICAMP), Sao Paulo-SP. Assistant \\ Professor, Department of Medicine, Center of Health Sciences, State University of Maringa (UEM), Maringa-PR, Brazil. Conception and design of \\ study, technical procedures, acquisition and analysis of data, manuscript writing. \\ IIPhD, Full Professor, Medical School, Pontifical Catholic University of Parana (PUCPR) and Associate Professor, Federal University of Parana \\ (UFPR), Curitiba-PR, Brazil. Histological and morphometric analysis. \\ IIIPhD, Associate Professor, Department of Pharmacology and Therapeutics, Center of Health Sciences, UEM, Maringa-PR, Brazil. Analysis of data. \\ ${ }^{\mathrm{IV}} \mathrm{PhD}$, Full Professor, Department of Surgery, Faculty of Medical Sciences, UNICAMP, Sao Paulo-SP, Brazil. Analysis of data, manuscript writing, \\ critical revision.
}

\begin{abstract}
PURPOSE: To study the repair of pericranium-cutaneous flaps fixed with suture anchored in a skull bone tunnel or N-butyl-2cyanoacrylate adhesive in Wistar rats with emphasis on the cellular inflammatory response and the production of types I and III collagen. METHODS: The operated region in the cephalic region of Wistar rats was removed minutes before euthanasia, fixed in formalin, and subjected to histological preparation. Slides were stained with hematoxylin-eosin and Picrosirius. Standardized counts of polymorphonuclear and mononuclear cells, fibroblasts, and macrophages were performed, and the percentages of types I and III collagen were determined. Data collection occurred on days $3,7,14,21$, and 45 postoperatively. A value of $\mathrm{p}<0.05$ was considered statistically significant.

RESULTS: Quantitative analysis of the data showed more fibroblasts in the surgical adhesive group than in the nylon monofilament thread groups $(\mathrm{p}=0.0211$ ). Qualitative analysis showed higher reactivity in the adhesive group, with a predominance of polymorphonuclear cells from days 3-45 and macrophages from days 3-7. The amount of type I collagen exceeded $80 \%$ in the treated and control groups at the end of the experiment.
\end{abstract}

CONCLUSIONS: Subperiosteal detachment triggers a cellular inflammatory response that is amplified using soft tissue fixation methods. The adhesive n-butyl-2-cyanoacrylate was more reactive than the nylon monofilament thread anchored in the skull bone tunnel.

Key words: Rhytidoplasty. Cyanoacrylates. Adhesives. Nylons. Wound Healing. Rats. 


\section{Introduction}

Traction and fixation of soft tissue flaps in facial plastic surgery is performed by means of soft tissue fixation methods, the most common being sutures, screws (metallic or absorbable), and surgical adhesives ${ }^{1-20}$.

Cyanoacrylates are cyanoacrylic acid esters with adhesive properties that polymerize at room temperature without a solvent or catalyst and that have one alkyl side chain in their molecular structure. Long chains confer less toxicity than do short chains. Cyanoacrylates thus demonstrate slow degradation in cyanoacetate and formaldehyde, limiting the accumulation of toxic byproducts that must be eliminated by the organism ${ }^{8-12}$.

Nylon monofilament threads are traditionally used as surgical sutures in soft tissues and are often compared with surgical adhesives ${ }^{13-15}$.

This work aimed to study the repair of pericraniumcutaneous flaps pulled and fixed with a nylon monofilament thread anchored in the skull bone tunnel or with a surgical adhesive, in Wistar rats. The histological tissue reactivity and production of types I and III collagen were also evaluated.

\section{Methods}

This study was approved under protocol number 018/2010 by the Ethics in the Use of Animal Experimentation Committee of the State University of Maringa (Brazil).

Thirty-five male Wistar rats (Rattus norvegicus albinus) at 120 days of age and weighing 280 to $484 \mathrm{~g}$ (average, $365.7 \mathrm{~g}$ ) were used. The rats were randomly distributed into three groups of ten animals as follows: Group A, control; Group B, suture anchored in a skull bone tunnel; and Group C, surgical adhesive. The last group of five animals constituted the Sham group (surgical control).

Beginning 24 hours prior to the experiments, the animals were acclimatized in a vivarium and maintained in plastic cages containing a maximum of four animals each. The rats received water and food ad libitum and were maintained at room temperature with a 12-h light/dark cycle.

After weighing the animals, the experimental procedure was initiated with intramuscular anesthesia using chlorhydrate 2-(2,6-xylidine)-5,6-dihydro-4H-1,3-thiazine (Rompun 2\%; Bayer, Sao Paulo-SP, Brazil) at a dose of $1 \mathrm{mg} / \mathrm{kg}$ and ketamine chlorhydrate (Ketalar; Pfizer, Guarulhos-SP, Brazil) at a dose of $20 \mathrm{mg} / \mathrm{kg}$ in a 1:1 ratio. Based on the dose of each drug, the mixture was used for anesthesia at a dose of $1 \mathrm{ml} / \mathrm{kg}$ of body weight.
With the animal anesthetized, the head was shaved followed by antisepsis of the shaved skin with an alcohol solution including 0.5\% chlorhexidine (Riohex; Rioquimica, Sao Jose do Rio Preto-SP, Brazil).

A 5 to 10-mm 3-0steel thread (Aciflex; Ethicon, Sao Paulo-SP, Brazil) was then introduced into the subcutaneous tissue as a dynamic radiological marker to observe the mobility of the flap during the study period. After introducing the radiological marker, the cranium of the animal was immobilized in a cephalostat to permit preoperative radiographs.

A 15-mm transverse cutaneous incision corresponding to the posterior border of the auricular pavilion was created. The incision allowed access to the deep planes and subsequent incision of the periosteum and thus the pericranium.

The subperiosteal flap was detached from the skull using a Freer elevator. The nasal dorsum and lateral orbits of the animal were the distal limits. Next, the elevated flap was suspended with the help of an Adson nipper. At this stage, the experimental model was ready for evaluation of the various fixation methods.

Group A underwent detachment and elevation of the flap without traction. Group B underwent the creation of two skull orifices $4 \mathrm{~mm}$ apart using a 2-mm round drill bit and preserving the duramater. A 4-0 nylon monofilament (Mononylon; Ethicon, Sao Paulo-SP, Brazil) thread was passed through from one opening to the other and anchored with a simple suture. The suture was also passed through the periosteum and subcutaneous tissue of the flap to promote and sustain traction on the flap (Figure 1).

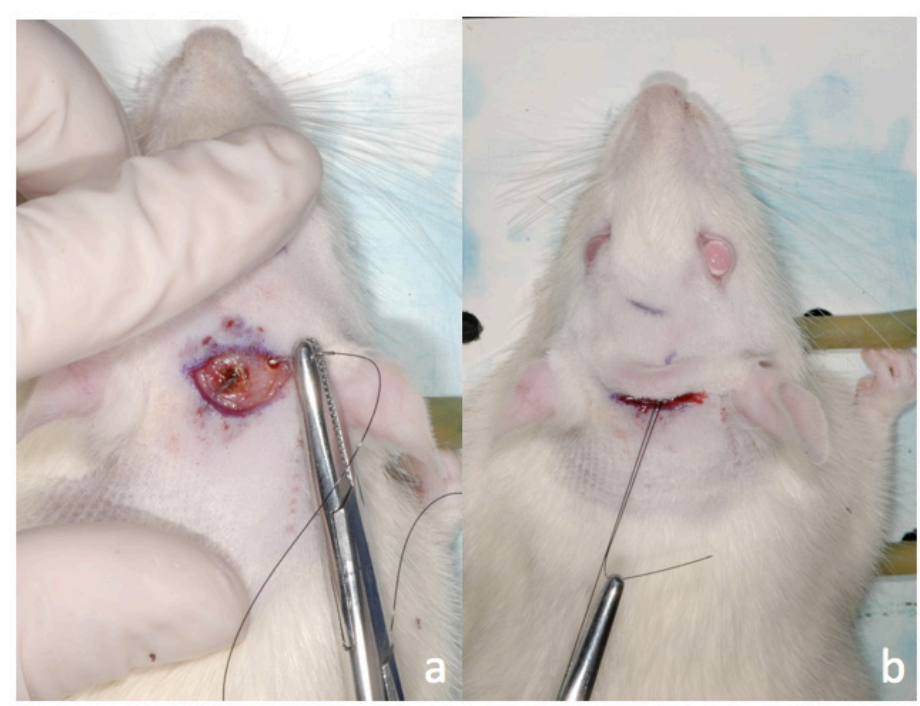

FIGURE 1 - (A) 4-0 nylon monofilament thread through the bone tunnel and (B) retracted flap. 
Group C underwent flap traction and received $0.25 \mathrm{ml}$ $(0.25 \mathrm{mg})$ per animal of n-butyl-2-cyanoacrylate surgical adhesive (Histoacryl 0.5g; B. Braun Melsungen AG, Melsungen, Germany) over the exposed bone of the skull using the actual ampule with an applicator tip (Figure 2).

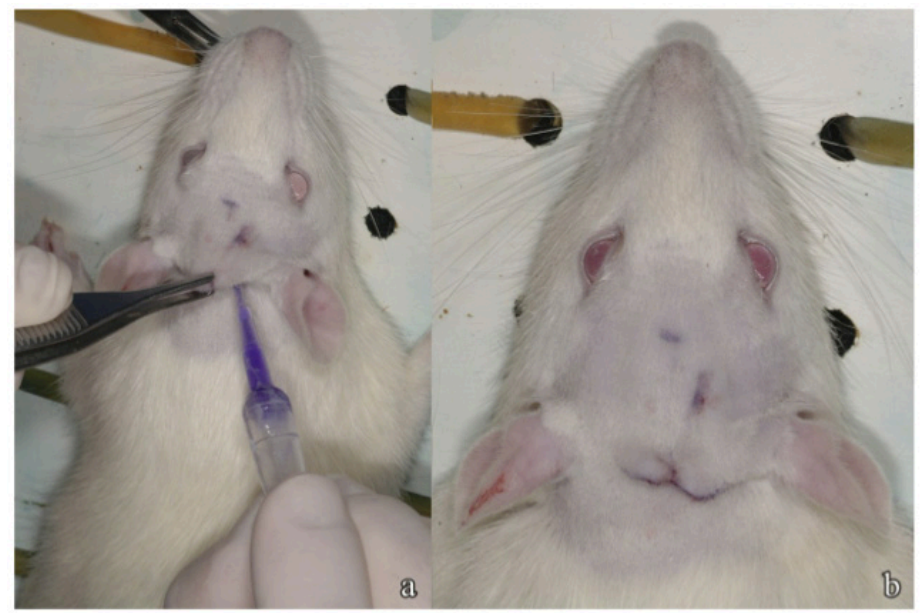

FIGURE 2 - (A) Application of n-butyl-2-cyanoacrylate glue and (B) retracted flap.

The skin incisions were sutured with three simple interrupted suturesof 5-0 monofilament nylon (Mononylon; Ethicon, Sao Paulo-SP, Brazil).

Postoperative radiographs were taken immediately after the surgery, and control radiographs were taken on postoperative days $3,7,14,21$, and 45 .

The animals received a single oral dose of paracetamol solution (Tylenol; Janssen-Cilag, Sao Paulo-SP, Brazil) at a dose of $10 \mathrm{mg} / \mathrm{kg}$ by gavage. Recovery occurred in the animals' cages.

Collection of tissue specimens for histological analysis was performed on days $3,7,14,21$, and 45 postoperatively after radiographs, even in anesthetized animals.

A skin incision edge bordering the upper eyelid allowed the removal of radiological marker not to damage the microtome during histological preparation. Proceeded another incision proximally located above the surgical incision and with the aid of a 2-mm round drill bit, there were four holes adjacent the proximal edge of the skull of the animal. Then two other incisions were made in the skin, bordering the edge of the eyelid contralateral arm, and the anterior border.

The four holes in the adjacent proximal edge of the skull facilitated introduction of Metzenbaum scissors to finish sectioning the bone along the path marked by the skin incisions to remove the specimen en bloc for histological study.

The material was fixed in formalin, and a central segment covering the greater extension of the operated region was embedded in paraffin after routine histological preparation. Histological sections $4 \mu$ thick were prepared on two slides for each animal, and then stained with hematoxylin-eosin and Picrosirius ${ }^{16}$.

The cellular inflammatory response was examined in the sections stained with hematoxylin-eosin. The same pathologist performed cell counts on five microscopic fields of $330 \mu \mathrm{m}^{2}$ corresponding to one sample from each animal in regions with a predominance of inflammatory cells adjacent to the pericranium at x400 magnification. Cells were sorted in terms of whether they were polymorphonuclear $(\mathrm{PMN})$ or mononuclear $(\mathrm{MN})$, and the numbers of fibroblasts (FIB) and macrophages (MAC) present in the microscopic fields were analyzed (Figure 3).

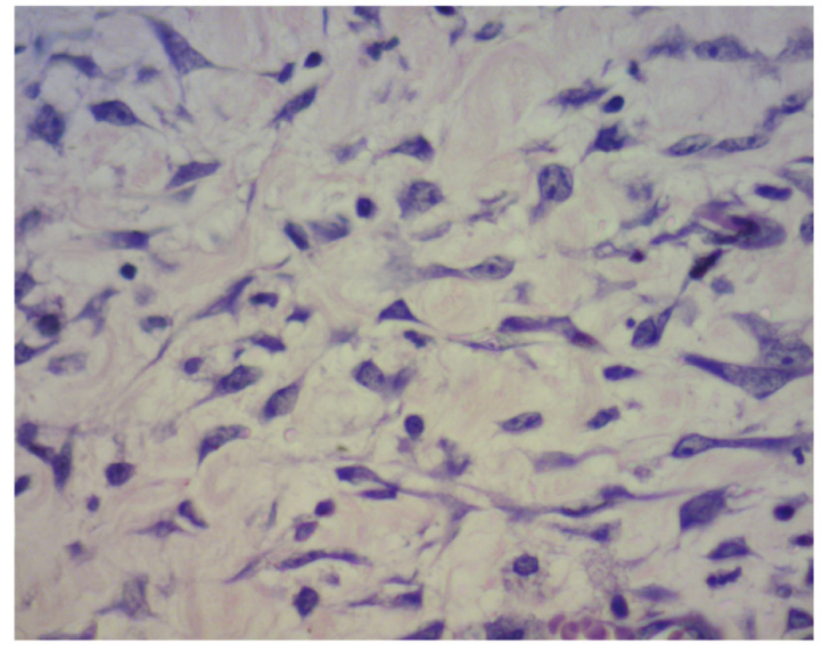

FIGURE 3 - Photomicrograph from Group C on postoperative day 7 (hematoxylin-eosin, x400 magnification).

Images of sections stained with Picrosirius were used to determine the percentages of types I and III collagen. Five different regions of each histological section were measured to obtain the average value of the collagen fibers present. The type I collagen fibers were thick and stained orange to red, and the type III collagen fibers were thin and stained green. Image-Pro Plus 4.0 software (Media Cybernetics Co., Roper Industries, Inc., Sarasota, FL, USA) allowed for the determination of the percentage of fibers of each color and enabled us to highlight them to better illustrate their tissue concentrations (Figure 4).

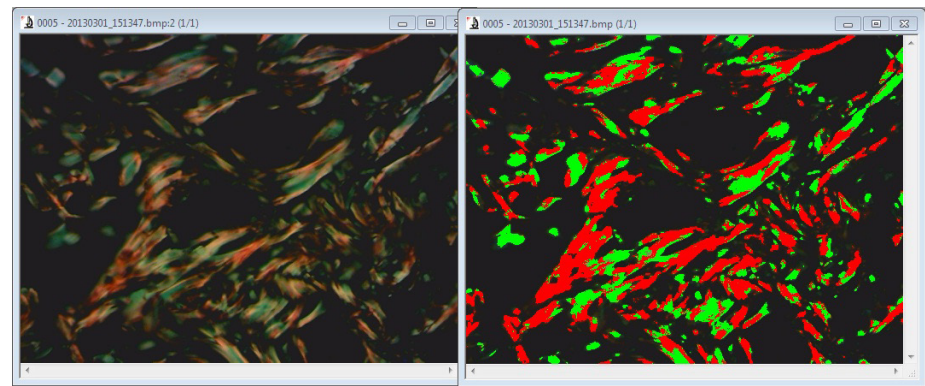

FIGURE 4 - Photomicrograph from Group B on postoperative day 7 with color highlighting of the fibers to the left (Sirius red, $\mathrm{x} 400$ magnification). 
Five digital photomicrographs of each rat and of each staining were obtained with a Dino-Eye camera AM423X (DinoLite, An Mo Electronics Corporation, New Taipei City, Taiwan).

Histological analysis was performed at the Laboratory of Experimental Pathology, Pontifical Catholic University of Parana in Curitiba, Brazil.

Quantitative data analysis, ANOVA analysis of variance, Tukey's test, and a T-test with a significance level of $5 \%$ were performed.

\section{Results}

Surgery with subperiosteal detachment led to an increase in the number of inflammatory cells at the surgical site. Both PMN and $\mathrm{MN}$ cells were present, the latter in larger quantities, but without a significant difference (Table 1).

TABLE 1 - Mean values of inflammatory polymorphonuclear $(\mathrm{PMN})$ and mononuclear (MN) cells in Groups Sham, A, B, and C at five time points.

\begin{tabular}{llrrrrr}
\hline \multirow{5}{*}{ Sham } & & Day 3 & Day 7 & Day 14 & Day 21 & Day 45 \\
& PMN & 0.2 & 0.2 & 0.2 & 0.2 & 0.2 \\
Group A & PMN & 1.8 & 2 & 2 & 0.8 & 2.2 \\
& MMN & 8.2 & 2.5 & 2.5 & 0.7 & 0.2 \\
Group B & PMN & 2.4 & 3.1 & 2.1 & 0 & 0 \\
& MMN & 4.4 & 4.6 & 5.8 & 1.5 & 0.8 \\
Group C & PMN & 3.4 & 3.6 & 2.5 & 1.5 & 0.2 \\
& MMN & 8 & 5.5 & 4.4 & 3.5 & 0.8 \\
\hline
\end{tabular}

* ANOVA significance level of 5\%

The qualitative data analysis demonstrated the effect of the surgery. An inflammatory response was triggered in Groups A, B, and $\mathrm{C}$; it peaked on postoperative day 7 and declined from postoperative day 14 onward. Throughout the repair process, there was a predominance of MN cells in Groups Sham, A, B, and C; however, from postoperative day 3 , the PMN count showed an inflammatory effect caused by the soft tissue fixation method used (suture anchored in the skull bone tunnel was less reactive than surgical adhesive). The $\mathrm{PMN}$ count remained higher in Group $\mathrm{C}$ from postoperative days $3-45$, than in the other groups (Figure 5).

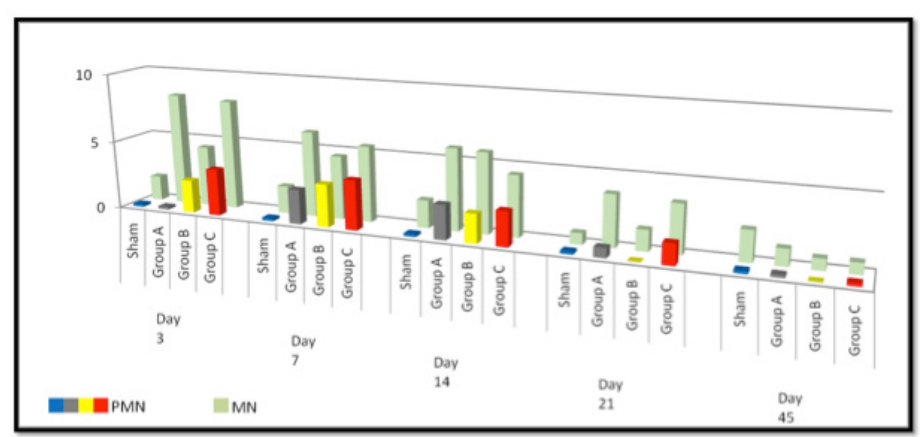

FIGURE 5 - Distribution of inflammatory polymorphonuclear (PMN) and mononuclear (MN) cells in Groups Sham, A, B, and C at five time points.
Subperiosteal detachment triggered tissue repair with fibroblast migration from postoperative days 3-7 and a decrease from postoperative day 14 on ward. This effect was more intense in Group C, which was treated with surgical adhesive (Table 2, Figure 6). There was a significant difference in the number of fibroblasts between Groups C and B on postoperative day 7 $(\mathrm{p}=0.0211)$ (Table 2, Figure 7).

TABLE 2 - Mean values of fibroblasts in Groups Sham, A, B, and $\mathrm{C}$ at five time points.

\begin{tabular}{lrrrrr}
\hline & Day 3 & Day 7 & Day 14 & Day 21 & Day 45 \\
Sham & 15 & 17 & 25.4 & 24 & 20.2 \\
Group A & 32.8 & 38 & 36.6 & 23.1 & 21.8 \\
Group B & 29.6 & $19.3^{* *}$ & 26.4 & 19.7 & 14.8 \\
Group C & 32 & $47.7^{* *}$ & 32.3 & 27.8 & 24.2 \\
\hline
\end{tabular}

* ANOVA significance level of $5 \%$

** T-test $\mathrm{p}=0,0211$

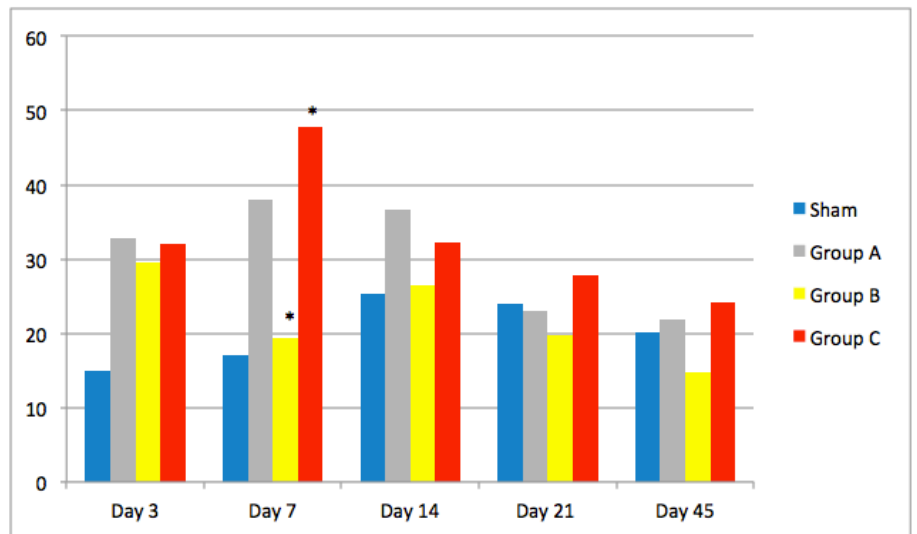

FIGURE 6 - Distribution of fibroblasts in Groups Sham, A, B, and C at five time points.

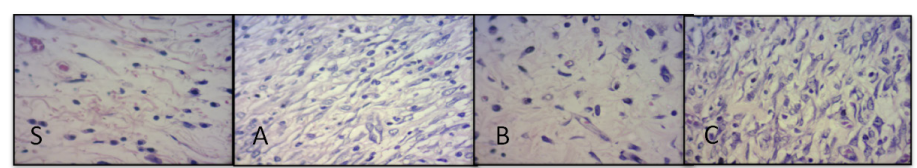

FIGURE 7- Photomicrographs from Groups Sham (S), A, B, and C on postoperative day 7 (hematoxylin-eosin staining, $\mathrm{x} 400$ magnification).

There was a predominance of macrophages in Group C on postoperative days 3 and 7 , but without statistical significance (Table 3).

TABLE 3 - Mean values of macrophages in Groups Sham, A, $\mathrm{B}$, and $\mathrm{C}$ at five time points.

\begin{tabular}{lrrrrr}
\hline & Day 3 & \multicolumn{1}{c}{ Day 7 } & Day 14 & Day 21 & Day 45 \\
Sham & 1.4 & 1 & 1 & 0.4 & 0.4 \\
Group A & 2.3 & 2.6 & 2.9 & 3 & 1.6 \\
Group B & 2.3 & 2.3 & 2.5 & 0.8 & 1.4 \\
Group C & 3.5 & 3.3 & 2.3 & 2.6 & 0.8 \\
\hline
\end{tabular}

*ANOVA significance level of $5 \%$ 
At the end of the experiment, there was a predominance of type I collagen fibers in the three operative groups without significant differences, but in excess of $80 \%$. This result highlights the role of subperiosteal detachment as a stimulus for repair (Figure 8).

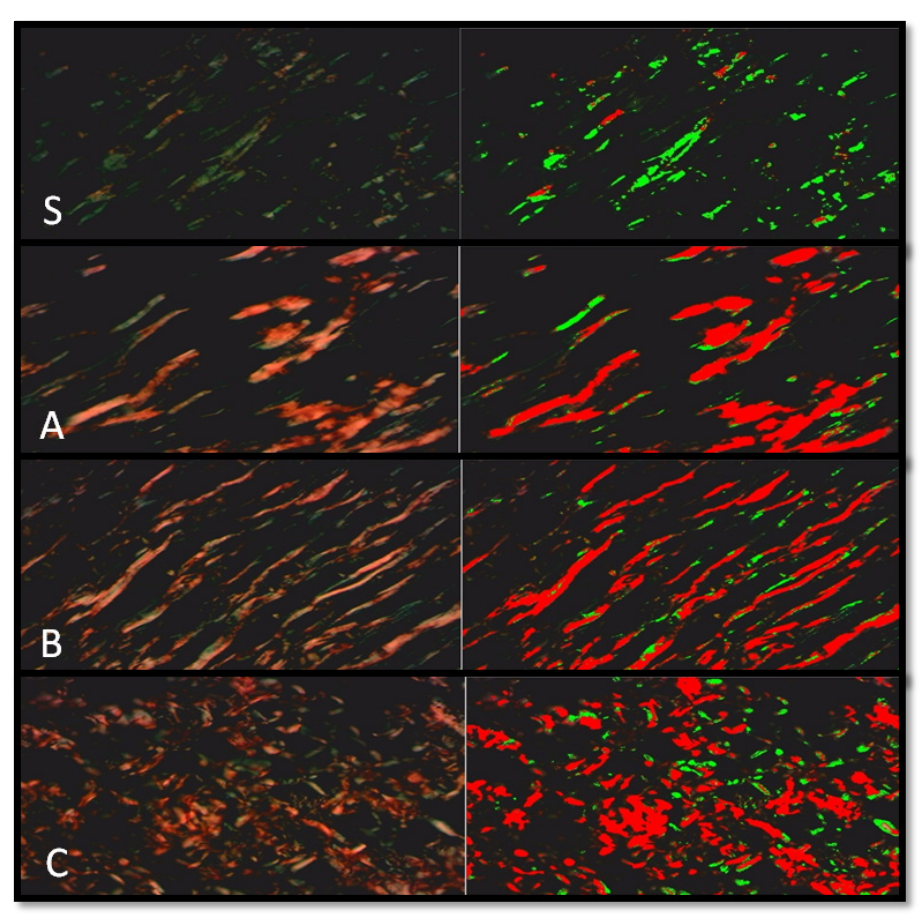

FIGURE 8 - Photomicrographs from Groups Sham (S), A, B and $\mathrm{C}$ on postoperative day 45 , with color highlighting of the fibers to the left (Sirius red, x400 magnification).

The surgical adhesive resulted in the highest collagen production at the end of the experiment in the operative groups to the order of $70.4 \%$ relative to the values on postoperative day 3 (Table 4, Figure 9).

TABLE 4 - Mean percentages of type I collagen in Groups Sham, A, B, and C at five time points.

\begin{tabular}{lrrrrr}
\hline & Day 3 & \multicolumn{1}{c}{ Day 7 } & Day 14 & Day 21 & Day 45 \\
Sham & 34,38 & 24,68 & 82,54 & 72,74 & 70,74 \\
Group A & 75,72 & 40,95 & 75,57 & 78,23 & 86,63 \\
Group B & 65,36 & 58,45 & 72,36 & 64,26 & 86,81 \\
Group C & 49,26 & 69,46 & 72,4 & 65,65 & 83,92 \\
\hline
\end{tabular}

* ANOVA significance level of $5 \%$

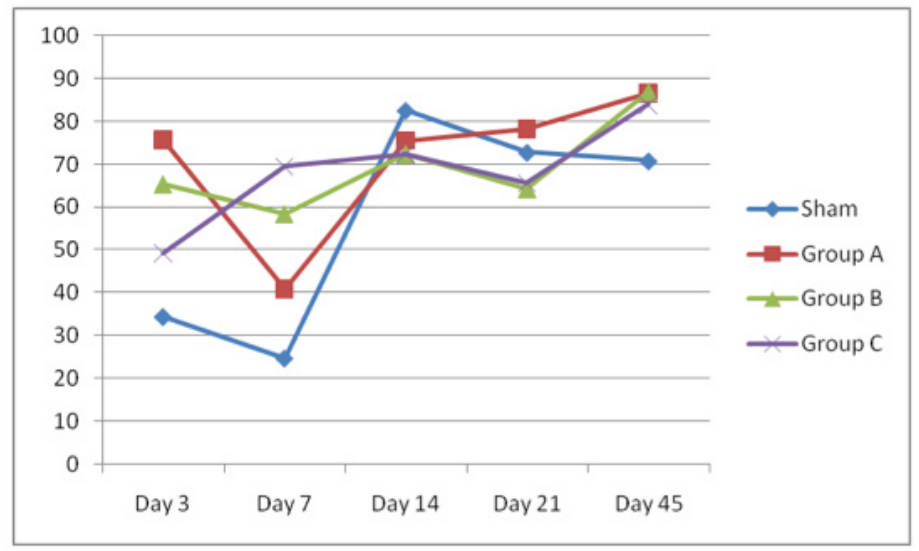

FIGURE 9 - Distribution of percentages of type I collagen in Groups Sham, A, B, and C at five time points.

\section{Discussion}

The study of tissue reactivity in surgery indirectly contributes to the prediction of results, so that measures taking and surgical planning, especially in plastic surgery. A successful outcome occurs in the proportion that the inflammatory response, which is individual, single, singular, manifests itself in a positive way for that individual, that is also unique, even within a group of the same species.

In this experimental study, subperiosteal detachment resulted in a more intense inflammatory response from postoperative day 3 in the groups that underwent flap fixation. The same effect was seen in Group A (control) from postoperative day 7. In all three operative groups, the inflammatory response declined on postoperative day 21.This demonstrates the need to perform displacements that promote wound healing, when repositioning the flap in the desired position. Likewise, the presence of soft tissue fixation methods increased the inflammatory reaction, thereby increasing collagen deposition and scar maturation. This was shown in the treated groups, in which the deposition of type I collagen was higher than that in the control group on postoperative day 7. Although the degree of collagen deposition was similar among the three groups at the end of the experiment, when the flap displacement was analyzed with a radiological control, we found the need to use soft tissue fixation methods to maintain the proposed tissue repositioning ${ }^{18}$.

The initial number in the study design of 15 animals in Group A and for each treated group was not approved by the Ethics in the Use of Animal Experimentation Committee, reducing the statistical significance.

Saltz and Zamora ${ }^{7}$ reported that endoscopic frontal rhytidectomy avoids a bicoronal incision. However, the ideal soft tissue fixation method was controversial and the authors 
reported the use of fibrin glue with good hemostatic function and for tissue fixing under a light pressure dressing. In the same year, Mixter ${ }^{19}$ reported the use of adhesive n-butyl-2cyanoacrylate in endoscopic frontal rhytidectomy as a safe and inexpensive fixative, and although he saw foreign body reaction in some patients, this resolved with the removal of the adhesive fragments. In our study, we emphasized the importance of developing an experimental model for the study of soft tissue fixation methods and their tissue reactions. We found that the adhesive n-butyl-2-cyanoacrylate is more reactive than suture anchored in a skull bone tunnel; thus, adhesive promotes increased migration of PMN cells and fibroblasts corresponding to the higher repositioning of the flaps in the long term ${ }^{18}$. We also observed during the collection of specimens for histological analysis the presence of cover slip adhesive polymerized between the soft tissue and the bone plate. This finding corresponded to that of Mixter ${ }^{19}$ and caused a chronic foreign body reaction. In our study, we found a larger amount of fibroblasts in Group C on postoperative days 3 and 7 , but an absence of multinucleated giant cells.

Mastieri et al. ${ }^{12}$ studied the inflammatory response of n-butyl-2-cyanoacrylate implanted in the subcutaneous tissue of rats and observed no significant differences compared with controls, concluding that this adhesive is biocompatible. Mugali et al. ${ }^{14}$ showed that in rats, this adhesive causes less stimulation of TNF alpha and IL-1beta than does catgut; it is also less immunogenic. We observed that the number of MN cells was high in all groups during the five days of measurement, but the humoral inflammatory response was not assessed.

Batista et al. ${ }^{15}$ compared sutures in the abdominal wall of rats that underwent operations using 3-0 nylon monofilament thread and adhesive n-butyl-2-cyanoacrylate. The suture time was faster in the adhesive group, and although there was one case of wound dehiscence and abscess formation, this group showed higher tensile strength than did the suture group on postoperative day 14 . In our study, we observed easy flap fixation and less surgical time when using adhesive and no occurrences of dehiscence or abscess formation. We believe that these reactions may be associated with the amount of adhesive applied.

Sadato et al..$^{20}$ studied the effect of dilutions of n-butyl2-cyanoacrylate/ethiodol in proportions of 20:80 and 50:50 for embolization of the renal arteries of rabbits and quantified the macrophages and neutrophils. No significant differences were seen throughout the study, nor were there differences in the degree of injury to the vascular wall. We believe that dose-control studies may be able to identify less reactive doses of n-butyl-2- cyanoacrylate. The development of devices with gradual adhesivereleasing capabilities or even less reactive adhesive isomers for use within soft tissues may also be possible.

\section{Conclusions}

Subperiosteal detachment triggers a cellular inflammatory response that is amplified when soft tissue fixation methods are used. The adhesive n-butyl-2-cyanoacrylate is more reactive than nylon monofilament thread anchored in a skull bone tunnel.

\section{References}

1. Honig JF, Frank MH, Knutti D, de La Fuente A. Video endoscopicassisted brow lift: comparison of the eyebrow position after endotine tissue fixation versus suture fixation. J Craniofac Surg. 2008;19:1140-7.

2. Citarella ER, Sterodimas A, Condé-Green A. Minimally invasive treatment of dynamic horizontal forehead lines. Plast Reconstr Surg. 2009;124(6):438e-9e.

4. Graf RM, Tolazzi ARD, Mansur AEC, Teixeira V, Knize DM. Endoscopic periosteal brow lift: evaluation and follow-up of eyebrow height. Plast Reconstr Surg. 2008;121:609-16.

5. Casagrande C, Saltz R, Chem R, Pinto R, Collares M. Direct needle fixation in endoscopic facial rejuvenation. Aesthetic Surg J. 2000;20:361-7.

6. Berkowitz RL, Apfelberg DB. Preliminary evaluation of a fastabsorbing multipoint fixation device. Aesthetic Surg J. 2008;28:584-8.

7. Saltz R, Zamora S. Tissue adhesives and applications in plastic and reconstructive surgery. Aesthetic Plast Surg. 1998;22:439-43.

8. Donkerwolcke M, Burny F, Muster D. Tissues and bone adhesiveshistorical aspects. Biomaterials. 1998;19:1461-6.

9. Fagundes DJ, Taha MO, Rivoire HC. Adesivos cirúrgicos: revisão e atualização. JBM. 2002;82:101-3.

10. Pani KC, Gladieux G, Brandes G, Kulkarni RK, Leonard F. The degradation of n-butyl alpha cyanoacrilate tissue adhesive. II. Surgery. 1968;63:481-9.

11. Saska S, Gaspar AM, Hochuli-Vieira E. Cyanoacrylate adhesives for the synthesis of soft tissue. An Bras Dermatol. 2009;84:585-92.

12. Mestieri LB, Saska S, Carrodeguas RG, Gaspar AM. Evaluation of n-butyl cyanoacrylate adhesive in rat subcutaneous tissue. Dermatol Surg. 2012;38:767-71.

13. Costa LA, Jardim PR, Macedo PH, Amaral VF, Silva AL, Barbosa CA. Evaluation of the abdominal wall cicatrization of rabbits exposed to nicotine and undergone abdominoplasty using nylon thread or cyanoacrylate. Acta Cir Bras. 2012;27:861-5.

14. Muglali M, Ylmaz N, Inal S, Guvenc T. Immunohistochemical comparison of indermil with traditional suture materials in dental surgery. J Craniofac Surg. 2011;22:1875-9.

15. Batista CA, Colleoni Neto R, Lopes Filho GJ. Comparative study of the healing process of the aponeurosis of the anterior abdominal wall of rats after wound closure using 3-0 nylon suture and N-butil2-cyanoacrylate tissue adhesive. Acta Cir Bras. 2008;23:352-63.

16. Junqueira LC, Bignolas G, Brentani RR. Picrosirius staining plus polarization microscopy, a specific method for collagen detection in tissue sections. Histochem J. 1979;11:447-55.

17. Troilius C. Subperiosteal brow-lift without fixation. Plast Reconstr Surg. 2004; 14:1595-603.

18. Cavazana WC, Cuman RK, Takeshita WM, Passeri LA. 
Experimental model for the study of soft tissuefixation methods on skin-pericranium flaps in rats. Acta Cir Bras. 2013;28:696-702.

19. Mixter RC. Endoscopic forehead fixation with histoacryl. Plast Reconstr Surg. 1998;101:2006-7.

20. Sadato A, Wakhloo AK, Hopkins LN. Effects of a mixture of a low concentration of n-butylcyanoacrylate and ethiodol on tissue reactions and the permanence of arterial occlusion after embolization. Neurosurgery. 2000;47:1197-203.

\section{Acknowledgement}

To biologist Seigo Nagashima, Laboratory of Experimental Pathology, Pontifical Catholic University of Parana the photomicrographs and collagen analysis.

\section{Correspondence:}

William César Cavazana

Universidade Estadual de Maringá

Departamento de Medicina

Avenida Mandacaru, 1590 - Bloco S05/Sala 10

87080-000 Maringá - PR Brasil

Tel.: (55 44)3262-0904

wccavazana@uem.br

Received: Dez 11, 2013

Review: Feb 13, 2014

Accepted: March 14, 2014

Conflict of interest: none

Financial source: Foundation for the Support of Scientific Development (FADEC)

${ }^{1}$ Research performed at Operative Technique, Experimental Surgery, Metabology and Plastic Surgery, Department of Medicine, Center of Health Sciences, State University of Maringa (UEM) and Pathology, Medical School, Pontifical Catholic University of Parana (PUC-PR), Curitiba-PR, Brazil. Part of PhD degree thesis, Postgraduate Program in Surgery Sciences, Faculty of Medical Sciences, State University of Campinas (UNICAMP). Tutor: Luis Augusto Passeri. 